\title{
Elevated Aurora Kinase A Protein Expression in Diabetic Skin Tissue
}

\author{
Moon Kyun Cho ${ }^{1}$, Je Min An ${ }^{1}$, Chul Han Kim², Sang Gue Kang ${ }^{2}$ \\ Departments of ${ }^{1}$ Dermatology and ${ }^{2}$ Plastic and Reconstructive Surgery, Soonchunhyang University College of Medicine, Seoul, Korea
}

Background Aurora kinase A (Aurora-A) plays an important role in the regulation of mitosis and cytokinesis. Dysregulated Aurora-A leads to mitotic faults and results in pathological conditions. No studies on Aurora-A expression in human diabetic skin tissue have been reported. In light of this, we explored the expression of Aurora-A in human diabetic skin tissue.

Methods Aurora-A protein was evaluated by western blotting in 6 human diabetic skin tissue and 6 normal skin specimens.

Results Increased expression of Aurora-A protein was detected in all diabetic skin tissue samples in both western blot analysis and immunohistochemical staining. However, in the case of the normal skin tissue, no bands of Aurora-A protein were detected in either the western blotting analysis or the immunohistochemical staining.

Conclusions Thus far, there have been no studies on the expression of Aurora-A in diabetic skin tissue. However, we believe that oxidative DNA damage related to the expression of Aurora-A protein and Aurora-A could be involved inhuman diabetic skin tissue.

Keywords Skin / Diabetes mellitus
Correspondence: Chul Han Kim Department of Plastic and Reconstructive Surgery, Soonchunhyang University College of Medicine, 59 Daesagwan-ro, Yongsan-gu, Seoul 140-743, Korea Tel: $+82-2-709-9283$

Fax: +82-2-796-3543

E-mail:kchann@hanmail.net

This work was supported by the Soonchunhyang University Research Fund.

No potential conflict of interest relevant to this article was reported.

Received: 30 May 2013 • Revised: 19 Aug 2013 • Accepted: 2 Sep 2013

pISSN: 2234-6163 • elSSN: 2234-6171 • http://dx.doi.org/10.5999/aps.2014.41.1.35 • Arch Plast Surg 2014;41:35-39

\section{INTRODUCTION}

Aurora kinases belong to the family of serine/threonine protein kinases, which are important in cellular proliferation [1]. Aurora kinases are involved in the control of the mitotic spindle, centrosome separation, centrosome duplication, chromosomal orientation, and the spindle assembly checkpoint as well as cytokinesis [2]. Hence, these Aurora kinases are considered to be the key regulators of mitosis. In humans, there are three classes of Aurora kinases, namely Aurora kinase A (Aurora-A), Aurora kinase B (Aurora-B), and Aurora kinase C (Aurora-C). They have a high homologous similarity in the amino acid chain. Aurora-A and Aurora-B are expressed in most types of normal cells. Aurora-C is highly expressed in the testis [3]. Of these Aurora kinases, Aurora-A has been thoroughly studied. Aurora-
A is also known as Aurora, Aurora A, Aurora-2, aurora/IPL1related kinase (AIK), aurora-related kinase 1 (ARK1), aurora A (AURA), AYK1, Breast tumour-amplified kinase (BTAK), Eg2, MmIAK1, serine/threonine kinase (STK6), STK7, STK15, AURORA2, and MGC34538. Aurora-A has important influences on the beginning of mitosis and cytokinesis. Further, dysregulated Aurora-A results in centrosomal defects, spindle assembly checkpoint faults, genetic imbalance, transformation, and neoplasm formation [4].

Diabetes mellitus is a serious disease that is widespread across the world. The pathogenesis and the complications of diabetes arise from various pathologic mechanisms including oxidative stress [5]. According to Brownlee [6], oxidative stress plays a critical role in tissue damage related to diabetes. Hsieh et al. [7] reported that oxidative stress causes DNA damage in diabetic 
rats. Recently, Bhatia et al. [8] reported that DNA damage targets Aurora-A. Although Aurora- $A$ is known to have oncogenic properties, thus far, no studies on the expression of Aurora-A in human diabetic skin tissue have been reported. Therefore, the author has investigated the expression of Aurora- $\mathrm{A}$ in both normal skin and human diabetic skin tissue in order to reveal the relationship between Aurora- $A$ and human diabetic skin tissue.

\section{METHODS}

The protocol of this study was reviewed and approved by the Institutional Review Board of Seoul Soonchunhyang University Hospital, including the use of tissue samples.

\section{Cell lines and tissue samples}

The human malignant melanoma cell line G361 (CRL 1424, Rockville, MD, USA) was obtained from the American Type Culture Collection. The cells were cultured in DMEM, 10\% FCS, $100 \mathrm{U} / \mathrm{mL}$ penicillin, and $100 \mathrm{mg} / \mathrm{mL}$ streptomycin at $37^{\circ} \mathrm{C}, 5 \% \mathrm{CO}_{2}$.

For the study, 6 normal skin tissue samples and 6 diabetic skin tissue samples were obtained from patients who underwent surgery between December 2012 and February 2013 in the Department of Plastic and Reconstructive Surgery at Soonchunhyang University Hospital in Korea. Informed consent was obtained from the patients before surgery. The normal skin tissue samples were collected from the lower leg of patients who had undergone flap reconstruction because of trauma; there patientshad no underlying medical disease. Diabetic skin tissue samples were obtained from patients undergoing amputation surgery, and normal skin tissue samples, which did not include necrosis, inflammation, gangrene, or ulcer, were harvested from these patients (Table $1)$. The specimens were frozen in liquid nitrogen immediately after resection and stored at $-80^{\circ} \mathrm{C}$. The stored formalin-fixed, paraffin-embedded samples were used for the study.

\section{Western blot analysis}

The human malignant melanoma cell line G361 served as a positive control for Aurora-A expression. Tissue samples were homogenized in a WCE buffer (25-mM HEPES [pH 7.7], 0.3$\mathrm{M} \mathrm{NaCl}, 1.5-\mathrm{mM} \mathrm{MgCl}_{2}, 0.2-\mathrm{mM}$ ethylenediamine tetraacetic acid EDTA, 0.1\% Triton X-100, 0.5 mM dithiothreitol DTT, 20 $\mathrm{mM}$ glycerolphosphate, $0.1-\mathrm{mM} \mathrm{Na}_{3} \mathrm{VO} 4,2 \mathrm{~g} / \mathrm{mL}$ leupeptin, 2 $\mathrm{g} / \mathrm{mL}$ aprotinin, $1 \mathrm{mM}$ phenylmethylsulfonyl fluoride, and a protease inhibitor cocktail tablet [Complete, Boehringer Mannheim $\mathrm{GmBH}$, Mannheim, Germany]). The tissue suspension was rotated at $4^{\circ} \mathrm{C}$ for 10 minutes. The supernatants were collected, kept at $-70^{\circ} \mathrm{C}$, and used for western blotting. Proteins from the tissue were separated by sodium dodecyl sulfate polyacrylamide gel electrophoresis (SDS-PAGE) using NuPAGE $4 \%$ to $12 \%$ bisTris gels (NuPAGE, NP0335Box, Invitrogen, Seoul, Korea) and then transferred to a polyvinyl difluoride membrane (Immobilon-P, Millipore Korea Co. Ltd., Seoul, Korea). The membrane was blocked using 5\% Bovine Serum Albumin (BSA) in TBST (20 mMTris, pH 7.6, $130 \mathrm{mMNaCl}$, and 0.1\% Tween 20) solution. Then, it was reacted with the primary antibody, Aurora-A rabbit polyclonal antibody (Cell SignalingTechnology, Catalog number 3092) diluted to a $1: 1,000$ concentration, stored at $4^{\circ} \mathrm{C}$ for 16 hours, and washed well with a washing buffer and a TrisBuffered Saline/TWEEN-20 (TBST) buffer (10-mM Tris$\mathrm{Cl}, \mathrm{pH}$ 8.0, $150-\mathrm{mM} \mathrm{NaCl}, 0.05 \%$ Tween 20) four times for 10 minutes, 10 minutes, 15 minutes, and 15 minutes, respectively, and reacted with anti-rabbit IgG (Cell Signaling Technology, Catalog number 7074)horseradish peroxidase-linked speciesspecific whole antibody diluted to 1:2,000 for 1 hour. After the reaction with the secondary antibody, it was washed well four times for 10 minutes, 10 minutes, 15 minutes, and 15 minutes, respectively. Proteins on the membrane were detected using the enhanced chemiluminescence solution kit (Amersham Pharmacia Biotech, Amersham, Buckinghamshire, UK). The membranes were stripped and reblotted with an anti-actin antibody (Sigma, St Louis, MI, USA; Catalog number A5441).

\section{Assessment of western blot analysis}

The relative abundance of each protein expression was analyzed using Phosphor-Imager software (TINA, ver. 2.10e, Raytest, Straubenhardt, Germany). Further, the measured scores of the expression of normal skin and diabetic skin tissues were compared.

\section{Table 1. Clinical characteristics of diabetic patients}

\begin{tabular}{|c|c|c|c|c|c|}
\hline Sex/Age (yr) & $\begin{array}{l}\text { Body mass index } \\
\left(\mathrm{kg} / \mathrm{m}^{2}\right)\end{array}$ & $\begin{array}{c}\text { Duration of diabetes } \\
(y r)\end{array}$ & Harvest skin site & Proteinuria & $\begin{array}{c}\text { Hemoglobin A1c } \\
(\%)\end{array}$ \\
\hline $\mathrm{M} / 71$ & 19.47 & 25 & Plantar skin of foot & ++ & 6.0 \\
\hline $\mathrm{M} / 80$ & 25.52 & 34 & Dorsal skin of foot & + & 7.4 \\
\hline $\mathrm{M} / 91$ & 21.19 & 6 & Dorsal skin of foot & - & 11.5 \\
\hline $\mathrm{M} / 79$ & 24.68 & 32 & Plantar skin of foot & + & 10.6 \\
\hline $\mathrm{F} / 76$ & 23.32 & 35 & Anterior skin of tibia & - & 8.6 \\
\hline $\mathrm{F} / 60$ & 19.77 & 27 & Dorsal skin of foot & ++ & 9.9 \\
\hline
\end{tabular}




\section{Immunohistochemical staining}

The stored formalin-fixed, paraffin-embedded samples, including 6 normal skin tissue samples and 6 diabetic skin tissue samples, were used in this test. The paraffin sections were deparaffinized in xylene, rehydrated in a 10-mM citrate buffer ( $\mathrm{pH}$ 6.0), and heated in a microwave oven for 15 minutes to restore the antigens. To suppress endogenous peroxidase within the tissues, the samples were treated with 3\% peroxide for 5 minutes and then with a blocking solution for 30 minutes. The slides were incubated with the primary Aurora-A antibody (Abcam Inc., Catalog number ab13824, Cambridge, UK) in a humid chamber for 60 minutes. Tissue staining was visualized with a 3,3'-diaminobenzidine (ScyTek, Logan, UT, USA) substrate chromogen solution.

\section{Assessment of immunohistochemical staining}

In the immunohistochemical evaluation, the histochemical score (HSCORE) was used for comparison and standardization [9]. The HSCORE of Aurora-A was determined by two independent investigators. In the Aurora-A stain, cells with brown cytoplasm under an optical microscopeindicate a positive result. The HSCORE was calculated using the following equation: HSCORE $\Sigma \mathrm{Pi}(\mathrm{i}+1)$, where i denotes the intensity of staining with a value of 0,1 , or 2 ( 0 , weakstaining; 1 , moderate staining; or 2 , strong staining) and Pi represents the percentage of stained cells varying from $0 \%$ to $100 \%(0.0-1.00)$. HSCOREs ranged from a minimum of zero in cases with no staining to a maximum of 3.0 in cases in which all of the cells were stained with maxi-

\section{Fig. 1. Western blot analysis of Aurora-A protein}

Aurora-A protein was over expressed at the protein level in diabetic skin tissue as determined by western blot analysis. The human malignant melanoma cell line G361 served as a positive control for AuroraA expression. The same membrane was reprobed with anti- $B$-actin as a loading control. Aurora-A, Aurora kinase $A ; N$, normal skin tissue; $D$, diabetic skin tissue.

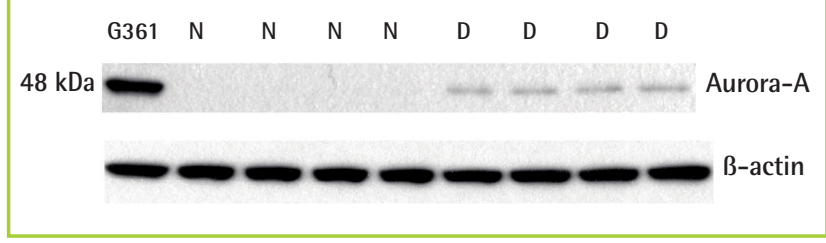

Table 2. HSCORE of Aurora-A expression in normal skin tissues and diabetic skin tissues

\begin{tabular}{|lccccc|}
\hline & $\mathbf{0}$ & $\mathbf{1 +}$ & $\mathbf{2 +}$ & $\mathbf{3 +}$ & Mean HSCORE \\
\hline Normal & 6 & 0 & 0 & 0 & 0.01 \\
Diabetic skin & 0 & 4 & 2 & 0 & 0.84 \\
\hline HSCORE, histochemical score.
\end{tabular}

mal intensity. The percentage of stained cellswas calculated by counting the positively stained cells among 500 cells using a $10 \times 10$ grid at a magnification of 400 .

\section{Statistical analysis}

The data from the Raytest TINA software were analyzed using the nonparametric Mann-Whitney $\mathrm{U}$ test. A value of $\mathrm{P}<0.05$ was considered to be statistically significant.

\section{RESULTS}

\section{Western blot analysis}

The results of the western blot analysis indicated that Aurora-A proteins were increasingly expressed in all of the human diabetic skin tissue samples but not expressed in the normal skin tissue (Fig. 1). The expression amount score in western blotting was measured using Raytest TINA software, and the median of Aurora-A was 0.11 (range, 0.01-0.14) in normal skin and 0.42 (range, 0.36-0.45) in human diabetic skin tissue. The expression amount score based on western blotting was graphed (Fig. 2); it revealed a significant difference in the Aurora-A protein expression between the normal skin and the diabetic skin $(\mathrm{P}<0.05)$.

\section{Immunohistochemical examination}

The immunohistochemical study revealed that the staining pattern of Aurora- $A$ in human diabetic skin tissue correlated with the results of the western blot analysis. Aurora-A protein was expressed in human diabetic skin tissue andwas stained at the basal cell layer. It was observed that inflammatory cells

\section{Fig. 2. Score of relative Aurora-A protein expression}

The median of Aurora kinase A (Aurora-A) was 0.11 (range, 0.01-0.14) in the normal skin, and the median of the diabetic skin tissue samples was 0.42 (range, 0.36-0.45). This revealed a significant difference in protein expression between the normal skin and diabetic skin $(P<0.05)$.

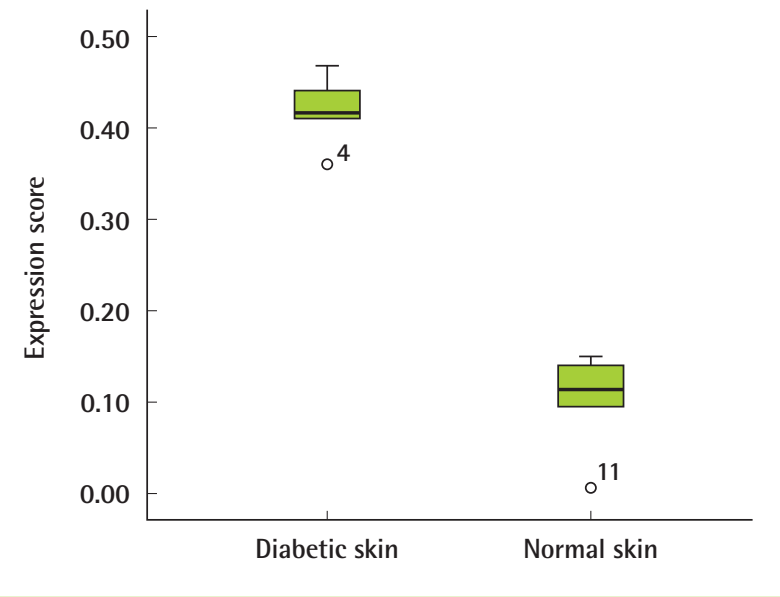




\section{Fig. 3. Immunochemical stain of Aurora-A protein expression}

(A) Aurora kinase A (Aurora-A) was not expressed in normal skin tissue (immunochemical stain, $\times 20)$. (B) Diabetic skin tissue displayed increased expression of Aurora-A (immunochemical stain, $\times 100$ ). Two black rectangle boxes show the magnified area. (C) Aurora-A was stained at the basal cell layer (black arrows) (immunochemical stain, $\times 400$ ). (D) It was observed that inflammatory cells (black arrow) were stained in the upper dermis (immunochemical stain, $\times 400)$.
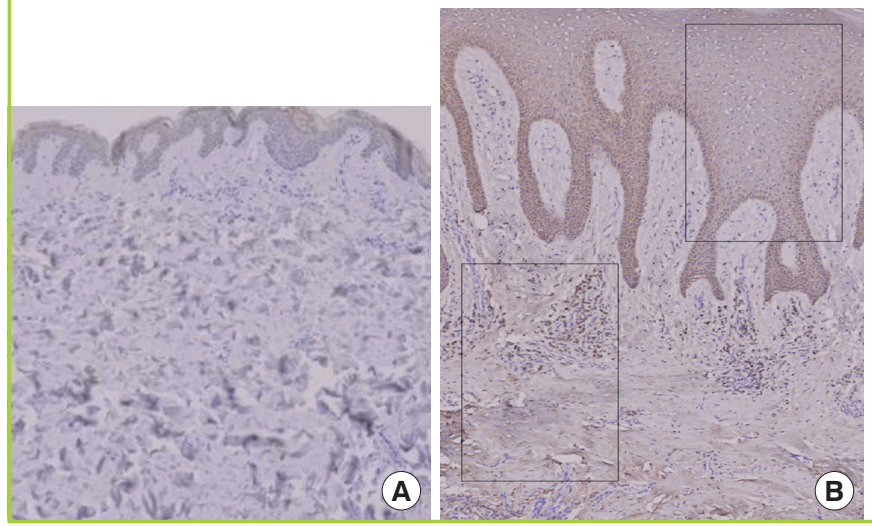

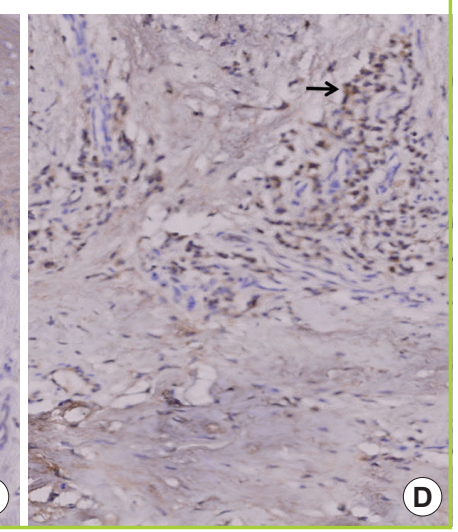

\section{Fig. 4. Relative intensity of Aurora-A expression}

The median of the normal skin tissue samples was 0.01 (range, $0.00-$ 0.03 ), and the median of the diabetic skin tissue was 0.85 (range, $0.78-$ 0.90). The expression of Aurora kinase A (Aurora-A) in the diabetic skin tissue was higher than in the normal tissues, and the difference was statistically significant (Mann-Whitney U test, $\mathrm{P}<0.05$ ).

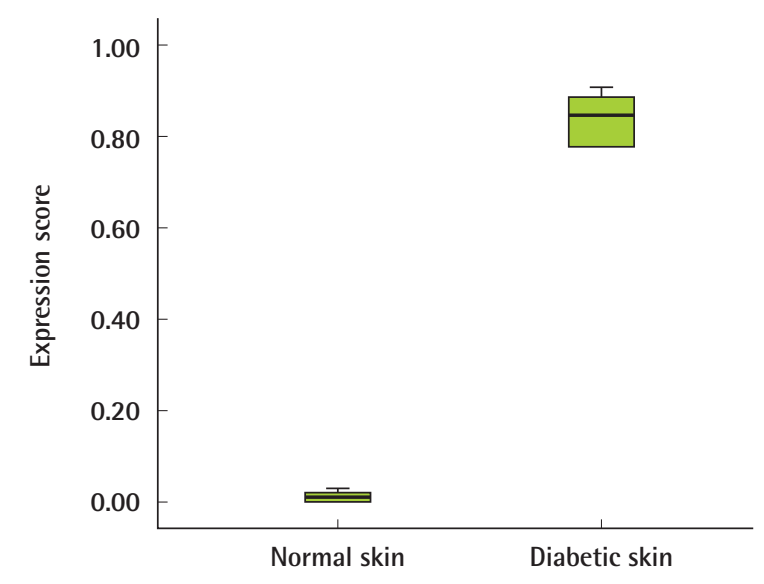

were stained in the upper dermis (Fig. 3). Aurora-A was not expressed in the normal skin tissue. In the normal skin tissue, the mean HSCORE was 0.01. In the diabetic skin tissue, the mean HSCORE was 0.84 (Table 2). The median of the normal skin tissue was 0.01 (range, 0.00-0.02), and the median of the diabetic skin tissue was 0.85 (range, 0.78-0.90). Aurora-A was expressed more intensively in the diabetic skin tissue, and the difference was statistically significant $(\mathrm{P}<0.05)$ (Fig. 4).

\section{DISCUSSION}

The Aurora kinase family consists of three different serine/threo- nine kinases, namely Aurora-A, Aurora-B, and Aurora-C. Aurora-A is related tocentrosome separation and spindle bipolarity $[10,11]$. Aurora-B is a chromosomal passenger protein and plays a certain role in chromosomal condensation, chromosomal congregation, chromosomal segregation, and cytokinesis [12]. Aurora-C, a chromosomal passenger protein similar to Aurora$B$, appears in the centrosome from anaphase to telophase and regulates mitotic chromosomal dynamics [3]. Aurora-A participates in cellular pathways and plays a vital role in the regulation of the core processes of mitosis and cytokinesis [13]. The Aurora-A gene is located within the chromosome locus 20q13.2. Further, Aurora-A is regulatedon the basis of the cell cycle [14]. Aurora-A is inactivated in the G1 phase of the cell cycle and is strongly expressed in the G2/M phases. A cell cannot function with a high level of Aurora kinase activity. Dysregulated AuroraA leads to mitotic faults and results in pathological conditions.

Diabetes mellitus is a type of metabolic disease accompanied by hyperglycemia, as well as lack of endogenous insulin secretion and activation. Recent studies have increasingly provided supporting evidence proving that both type 1 and type 2 diabetes mellitus are affected by oxidative stress $[5,6]$. Oxidative stress has been involved in pancreatic beta-cell damage, insulin resistance, and vascular dysfunction [5]. Glyco-oxidization, nonenzymatic glycation of protein, and subsequent oxidative degradation of glycated protein irregularly form free radicals. Overproduction of oxygen free radicals contributes to the progression of diabetes. Hsieh et al. [7] suggested that reactive oxygen species cause diabetes in the experimental rat model. They demonstrated that oxidative mitochondrial DNA damage occurred in multiple tissues of the liver, kidney, pancreas, brain, and heart of streptozotocin-induced diabetic rats [7]. DNA is susceptible 
to damage by reactive oxygen species, such as superoxide, hydrogen peroxide, hydroxyl radical, and singlet oxygen [15]. Not only mitochondrial damage and nuclear DNA damage but also the free radical-induced damage of the cell should be restored. Mitosis may be stopped by DNA damage. Mitosis is important for the regeneration of tissues and cellular integrity [16]. Hence, DNA damage by oxidative stress results in the blockade of mitosis andcan triggeran arrest before cell division.

DNA is normally regulated by many proteins that can identify the damage type and execute DNA repair [8]. With respect to such repairs, cells activate the signal mechanism to arrest the cell cycle [17]. Krystyniak et al. [18] demonstrated that DNA damage produced the arresting of the G2 transition in the cell cycle and accumulated the Aurora-A protein. Bhatia et al. [8] reported that mitotic DNA damage targeted Aurora-A. Further, a coincidence of the expression of elevated Aurora- $A$ and the suspension of a DNA damage-induced cell death response was observed in the study conducted by Katayama et al. [19]. These results led us to investigate the expression of Aurora-A protein in the skin tissue of diabetic patients.

Thus far, no studies on Aurora-A expression in human diabetic skin tissue have been reported. We used a western blot analysis method to examine the expression of Aurora-A in normal skin tissue and the skin tissue of diabetic patients. The results demonstrated that Aurora-A was found to be expressed more strongly in human diabetic skin tissue. Although there is no information on the exact mechanisms of Aurora-A protein expression thus far, we concluded that the oxidative DNA damage may have had some relationship to the expression of Aurora-A protein in diabetic skin tissue and that Aurora-A might be involved in diabetic symptoms in skin tissue. This study has some limitations: antagonists or siRNA may be analyzed for Aurora- $A$ to definethe potential involvement of Aurora-A in diabetic conditions. It will be necessary to conduct further research in order to verify as well as the role and mechanism, as well as the role and mechanism pathways of Aurora-A.

\section{REFERENCES}

1. Girdler F, Gascoigne KE, Eyers PA, et al. Validating Aurora B as an anti-cancer drug target. J Cell Sci 2006;119:3664-75.

2. Carmena M, Earnshaw WC. The cellular geography of aurora kinases. Nat Rev Mol Cell Biol 2003;4:842-54.

3. Li X, Sakashita G, Matsuzaki H, et al. Direct association with inner centromere protein (INCENP) activates the novel chromosomal passenger protein, Aurora-C. J Biol Chem 2004;279:47201-11.
4. Karthigeyan D, Prasad SB, Shandilya J, et al. Biology of Aurora A kinase: Implications in cancer manifestation and therapy. Med Res Rev 2010 Mar 1 [Epub]. http://dx.doi. org/10.1002/med.20203.

5. West IC. Radicals and oxidative stress in diabetes. Diabet Med 2000;17:171-80.

6. Brownlee M. Biochemistry and molecular cell biology of diabetic complications. Nature 2001;414:813-20.

7. Hsieh RH, Lien LM, Lin SH, et al. Alleviation of oxidative damage in multiple tissues in rats with streptozotocin-induced diabetes by rice bran oil supplementation. Ann N Y Acad Sci 2005; 1042:365-71.

8. Bhatia P, Menigatti M, Brocard M, et al. Mitotic DNA damage targets the Aurora A/TPX2 complex. Cell Cycle 2010; 9:4592-9.

9. Park HS, Park CH, Choi BR, et al. Expression of heat shock protein 105 and 70 in malignant melanoma and benign melanocytic nevi. J Cutan Pathol 2009;36:511-6.

10. Dutertre S, Descamps S, Prigent C. On the role of aurora-A in centrosome function. Oncogene 2002;21:6175-83.

11. Tsai MY, Wiese C, Cao K, et al. A Ran signalling pathway mediated by the mitotic kinase Aurora A in spindle assembly. Nat Cell Biol 2003;5:242-8.

12. Murata-Hori M, Tatsuka M, Wang YL. Probing the dynamics and functions of aurora B kinase in living cells during mitosis and cytokinesis. Mol Biol Cell 2002;13:1099-108.

13. Mortlock AA, Foote KM, Heron NM, et al. Discovery, synthesis, and in vivo activity of a new class of pyrazoloquinazolines as selective inhibitors of aurora B kinase. J Med Chem 2007;50:2213-24.

14. Lengauer C, Kinzler KW, Vogelstein B. Genetic instabilities in human cancers. Nature 1998;396:643-9.

15. Henle ES, Linn S. Formation, prevention, and repair of DNA damage by iron/hydrogen peroxide. J Biol Chem 1997;272: 19095-8.

16. Qi M, Yu W, Liu S, et al. Septin1, a new interaction partner for human serine/threonine kinase aurora-B. Biochem Biophys Res Commun 2005;336:994-1000.

17. Jackson SP, Bartek J. The DNA-damage response in human biology and disease. Nature 2009;461:1071-8.

18. Krystyniak A, Garcia-Echeverria C, Prigent C, et al. Inhibition of Aurora A in response to DNA damage. Oncogene 2006;25:338-48.

19. Katayama H, Wang J, Treekitkarnmongkol W, et al. Aurora kinase-A inactivates DNA damage-induced apoptosis and spindle assembly checkpoint response functions of $\mathrm{p} 73$. Cancer Cell 2012;21:196-211. 